$6-9-2020$

\title{
Idiopathic pulmonary hemosiderosis - A rare cause of chronic anemia
}

Ayesha Butt

Aga Khan University, ayesha.butt@scholar.aku.edu

Rashida Ahmed

Aga Khan University, rashida.ahmed@aku.edu

Muhammad Dawood Amir Sheikh

Aga Khan University

Omar Khan

Jinnah Sindh Medical University, Karach

Nousheen Iqbal

Aga Khan University, nousheen.iqbal@aku.edu

See next page for additional authors

Follow this and additional works at: https://ecommons.aku.edu/pakistan_fhs_mc_mc

Part of the Critical Care Commons, Pathology Commons, Pediatrics Commons, and the Pulmonology Commons

\section{Recommended Citation}

Butt, A., Ahmed, R., Sheikh, M. A., Khan, O., Iqbal, N., Rahman, A., Khan, J. (2020). Idiopathic pulmonary hemosiderosis - A rare cause of chronic anemia. Monaldi Archives for Chest Disease, 90(2).

Available at: https://ecommons.aku.edu/pakistan_fhs_mc_mc/128 


\section{Authors}

Ayesha Butt, Rashida Ahmed, Muhammad Dawood Amir Sheikh, Omar Khan, Nousheen Iqbal, Arshalooz Rahman, and Javaid Khan 


\title{
Idiopathic pulmonary hemosiderosis - A rare cause of chronic anemia
}

\author{
Ayesha Butt ${ }^{1}$, Rashida Ahmed ${ }^{2}$, Muhammad Dawood Amir Sheikh¹, Omar Khan ${ }^{3}$, Nousheen Iqbal ${ }^{3,4}$, \\ Arshalooz Jamila Rahman ${ }^{5}$, Javaid Ahmed Khan ${ }^{4}$ \\ ${ }^{1}$ Aga Khan University Medical College, Karachi; ${ }^{2}$ Section of Histopathology, Department of Pathology and Laboratory \\ Medicine, Aga Khan University, Karachi; ${ }^{3}$ Jinnah Sindh Medical University, Karachi; ${ }^{4}$ Department of Medicine, Section \\ of Pulmonary and Critical Care, Aga Khan University, Karachi; ${ }^{5}$ Department of Pediatrics and Child Health, Aga Khan \\ University, Karachi, Pakistan
}

\begin{abstract}
Idiopathic pulmonary hemosiderosis (IPH) is a rare disease marked by alveolar bleeding and accumulation of hemosiderin in the lungs. Here we present three cases of IPH. The first case is of 26-year-old male with anemia, hemoptysis and dyspnea. Bronchoscopy confirmed diffuse alveolar hemorrhage (DAH). A diagnosis of IPH was made after ruling out other causes of DAH and observing good response to steroids. The patient's condition
\end{abstract}

Correspondence: Ayesha Butt, Aga Khan University Medical College, G.407, Female Hostel, Aga Khan University Hospital, Stadium Road, Karachi, Pakistan. Karachi, Pakistan.

E- mail: ayeshabutt21@outlook.com

Key words: Hemoptysis; anemia; lung diseases.

Clonflict of interest: The authors declare no competing interests.

Authors' contributions: AB, contributed to design of the work; acquisition, analysis, interpretation of data, literature search, manuscript drafting and revision; RA, NI, contributed to conception and design of the work, and revising it critically for important intellectual content; MDAS, OK, contributed to acquisition, analysis, interpretation of data, manuscript drafting; AJR, JAK, contributed to conception and design of the work, revising it critically for important intellectual content. All the authors have read and approved the final version of the manuscript and agreed to be accountable for all aspects of the work.

Ethics approval and consent to participate: Obtained.

Consent for publication: Obtained.

Availability of data and material: Available from the corresponding author on reasonable request.

Received for publication: 8 March 2020

Accepted for publication: 24 April 2020.

${ }^{\circ}$ Copyright: the Author(s), 2020

Licensee PAGEPress, Italy

Monaldi Archives for Chest Disease 2020; 90:1267

doi: 10.4081/monaldi.2020.1267

This article is distributed under the terms of the Creative Commons Attribution Noncommercial License (by-nc 4.0) which permits any noncommercial use, distribution, and reproduction in any medium, provided the original author(s) and source are credited. improved with prednisolone and azathioprine. The second case is of 26-year-old female with severe anemia. Imaging suggested IPH and lung biopsy confirmed it. She died shortly afterwards. The third case is of a 7-year-old male with chronic anemia. CT was suggestive of IPH and lung biopsy confirmed the diagnosis. Later, patient developed posterior reversible encephalopathy syndrome (PRES). This patient is stable on azathioprine and prednisolone. We aim to emphasize the importance of considering IPH as a differential in patients with DAH or chronic anemia.

\section{Introduction}

Idiopathic pulmonary hemosiderosis (IPH) is a life-threatening parenchymal lung disease characterized by the destruction of pulmonary microvasculature, leakage of blood into the alveolar space and accumulation of hemosiderin in the lungs. It classically presents as a triad of hemoptysis, iron deficiency anemia and alveolar infiltrates. The condition is more commonly found in children with an approximate incidence of $0.24-1.23$ cases per million in different populations [1]. Only $20 \%$ of IPH cases occur in adulthood [2]. The rarity of the condition and the varying clinical course has made it difficult to diagnose this disease [3].

Here we present three cases of IPH presenting to our center, including two adult and one pediatric case. Through our report we aim to emphasize the importance of considering IPH as an important differential diagnosis when approaching a patient with diffuse alveolar hemorrhage or chronic anemia even in adults, given the potential morbidity and mortality of the disease.

\section{Case \#1}

A 26-year-old male admitted with complains of generalized weakness, hemoptysis and dyspnea was found to have a hemoglobin of $5.2 \mathrm{~g} / \mathrm{dl}$. Gastrointestinal endoscopy was performed to investigate the cause of anemia but revealed no bleeding source. The patient had been transfused 5 packs of blood. The patient was tachypneic (respiratory rate: $30 / \mathrm{min}$ ) and oxygen saturation of $75 \%$ on room air with bilateral crackles on chest auscultation Chest x-ray revealed bilateral alveolar infiltrates. High Resolution Computed Tomography (HRCT) chest delineated diffuse bilateral alveolar infiltrates (Figure 1). Bronchoscopy was done which showed blood throughout the airways and sequential bronchoalveolar lavage confirmed diffuse alveolar hemorrhage (DAH). Bronchoalveolar lavage (BAL) was negative for any microbiological organism or 
malignant cells. Lung biopsy was not done because of hypoxia. The patient had negative antinuclear antibody (ANA), antidouble stranded DNA (anti-dsDNA), Human Immunodeficiency Virus (HIV) and antineutrophilic cytoplasmic antibody (ANCA) tests. Sputum acidfast bacilli (AFB) smear, acid-fast bacilli (AFB) culture and GeneXpert MTB/RIF (diagnostic test that can identify Mycobacterium tuberculosis (MTB) DNA and resistance to rifampicin (RIF)) were negative for Mycobacterium tuberculosis. The patient was started on intravenous methylprednisolone, on which his condition improved. Considering his history and ruling out autoimmune and infectious etiologies and vasculitis, a diagnosis of IPH was made after exclusion of other causes of DAH and seeing the good response to steroid therapy. The patient's treatment regimen was later switched to $1 \mathrm{mg} / \mathrm{kg}$ oral prednisolone and $200 \mathrm{mg}$ ferrous sulfate. After discharge, the patient had a relapse of symptoms on a tapering dose of steroids and his hemoglobin level dropped back to $6.9 \mathrm{mg} / \mathrm{dl}$. Hence, the patient was restarted on prednisolone with addition of azathioprine. The patient responded well to this treatment. Within 6 weeks his symptoms resolved and his chest x-ray returned to normal.

\section{Case \#2}

A 26-year-old female presented with four years long history of cough along with scanty hemoptysis on and off. Her cough and dyspnea had worsened in the 3 months prior to presentation. She also gave history of recurrent anemia and multiple blood transfusions. On examination, her oxygen saturation was $86 \%$ on room air and she had bilateral fine crackles more marked at lower parts of chest. Her hemoglobin at presentation was $8.0 \mathrm{~g} / \mathrm{dl}$. Her Chest Xray and $\mathrm{CT}$ scan findings were suggestive of interstitial lung disease. She was transfused two units of packed cells and an open lung biopsy was done. Biopsy findings were consistent with IPH. This patient was started on steroids but she did not respond. She died few weeks later with respiratory failure secondary to hospital acquired pneumonia.

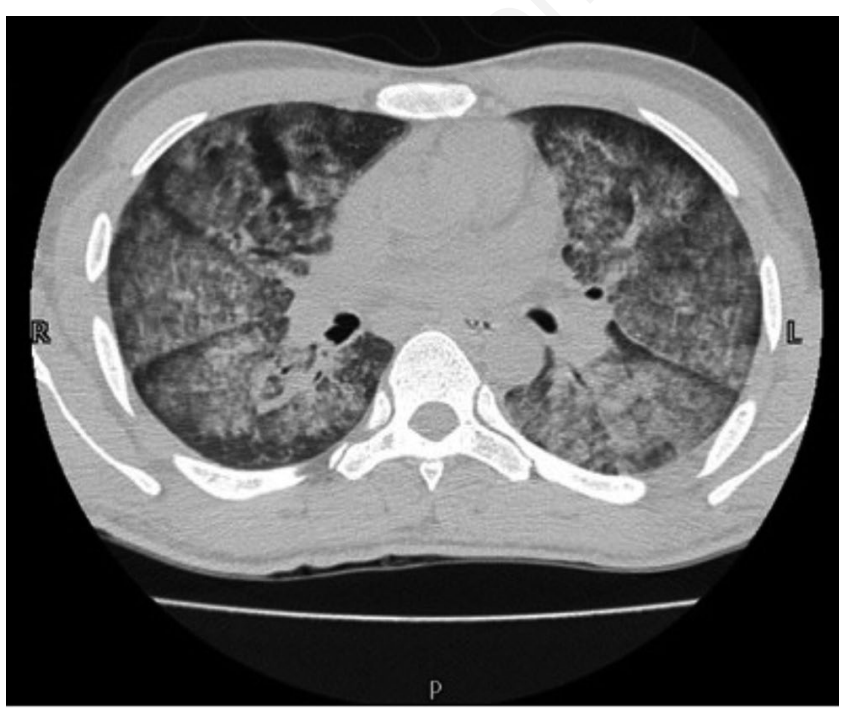

Figure 1. High resolution computed tomography (HRCT) scan chest of Patient 1 showing diffuse bilateral alveolar infiltrates.

\section{Case \#3}

A 7-year-old boy who had experienced shortness of breath and anemia for the past five years, presented for evaluation of chronic anemia. The patient had been transfused blood twice in the years before presenting to us.

At presentation, he had a hemoglobin level of $3.2 \mathrm{gm} / \mathrm{dl}$. The patient was transfused one packed cell unit of blood and thereafter bone trephine revealed absent storage iron on bone marrow, consistent with iron deficiency anemia. The child was then discharged on ferrous sulfate. After discharge, the patient had frequent hospital admissions due to cough and fever episodes. Then the patient was admitted again due to a five-day history of cough, fever respiratory distress and decreased oral intake. The patient was intubated and investigations revealed right sided pneumothorax for which chest tube was inserted. CT scan depicted evidence of diffuse ground glass haze in bilateral lung fields, which led to suspicion of pulmonary hemosiderosis and a subsequent lung biopsy confirmed the diagnosis (Figure $2 \mathrm{~A}, \mathrm{~B}$ ). The patient was started on prednisolone $10 \mathrm{mg}$ twice daily. The child was later hospitalized after he presented with multiple episodes of tonic clonic seizures. CT scan brain showed bilateral infarcts following a venous territory. Glascow Coma Scale score improved with time $(8 / 15$ at presentation to $15 / 15)$. However, the weakness on the right side persisted in both the upper and lower limbs. Magnetic resonance venography (MRV) was negative for any thrombus, whereas magnetic resonance imaging (MRI) brain showed multiple high signals in the cortex and white matter primarily in bilateral parieto-occipital and few areas in bilateral frontal lobes leading to a diagnosis of posterior reversible encephalopathy syndrome (PRES), possibly due to hypertension. ECHO and Doppler renal ultrasound, $24 \mathrm{~h}$ vanillylmandelic acid (VMA) and renin was normal. The patient was prescribed amlodipine, atenolol, azathioprine $37.5 \mathrm{mg}$ once daily, prednisolone $20 \mathrm{mg}$ twice daily and valproate sodium $400 \mathrm{mg}$ twice daily. The patient remains stable on the afore mentioned drugs ten years after his initial presentation to our center.

\section{Discussion}

Many different hypotheses have been postulated in an attempt to explain the etiology of IPH including allergic, environmental, auto immune and genetic causes. The allergic hypothesis arises from the frequent association between cow's milk allergy and IPH (Heiner syndrome) [4]. The environmental theory emerged after the development of IPH in children after exposure to Stachybotris chartarum [5]. IPH has been reported in siblings leading to the notion of an underlying genetic etiology. However, IPH has not been ascribed to a specific gene [6]. The auto-immune theory is corroborated by the frequent association of IPH with many different autoimmune diseases like immunoglobulin gammopathies, celiac disease, rheumatoid arthritis and glomerulonephritis [7]. Several studies have delineated that 1 in 4 children who survive IPH go on to develop auto immune diseases [8]. The positive influence of immunosuppressants in treatment of IPH further endorses the immunological theory. IPH accompanied by celiac disease is referred to as LaneHamilton syndrome. A gluten free diet has been reported to cause a dramatic amelioration in not only the symptoms of celiac disease but also those of IPH [9]. The French RespiRare cohort 
study further bolstered the notion of an autoimmune basis for IPH based on the findings that $68 \%$ of IPH patients had autoimmune antibodies at presentation, the most common being anti smooth muscle antibodies (50\%) and anti-nuclear antibodies $(45 \%)$. Moreover, celiac disease anti-bodies (anti-gliadin, antiendomysium and anti-transglutaminase antibodies) were present in $28 \%$ of patients. A surprising finding in the aforementioned study was the high prevalence $(20 \%)$ of Down syndrome in IPH patients whereas the incidence of Down syndrome in the French population is only $1 / 2000$ births annually. Down syndrome is also associated with a worse prognosis for IPH [10].

Patients who suffer from IPH generally show clinical findings of recurrent cough, shortness of breath and hemoptysis. Further investigations reveal the presence of iron deficiency anemia and pulmonary infiltrates attributed to alveolar capillary bleeding and elevated levels of hemosiderin in the lungs. The clinical course can vary from patient to patient, especially in children. A systematic review demonstrated that in adults the most common presentations were hemoptysis, dyspnea and anemia ( $81 \%, 62 \%$ and $54 \%$, respectively). In contrast, in children anemia may be the first and only finding, with hemoptysis being much less commonly encountered [11]. According to a study carried out in Romania, among the fifteen children diagnosed with IPH from 1984 to 2006, only 4 presented with the classical triad of IPH [11]. A cohort of 25 pediatric IPH cases delineated the most common presentations to be anemia and dyspnea $(64 \%$ and $68 \%$, respectively) with hemoptysis only reported in $50 \%$ of cases [12]. Amongst the patients described in our series as well, adults manifested the classical triad of IPH whereas the pediatric patient's first and main complaint was anemia, followed by dyspnea. A worrying factor is the delay in diagnosis of IPH which was seen in all our cases and has also been noted in various previous studies and ranges from 1-6.3 years [13]. The development of posterior reversible encephalopathy syndrome (PRES) in Case \#3 is a unique feature, with no associations between PRES and IPH reported other than in this patient.

Chest X-ray and computed tomography (CT) usually reveal nonspecific findings, making bronchoalveolar lavage (BAL) sig- nificant for the primary diagnosis of diffuse alveolar hemorrhage (DAH). During acute DAH, diffuse lung infiltrates are exhibited on CT chest and pulmonary function tests (PFTs) show increased diffusion capacity for carbon monoxide, which is strongly suggestive of alveolar hemorrhage. A diagnosis of IPH can only be finalized after excluding all other possible causes of DAH because IPH does not have pathognomonic findings. Generally, bland alveolar hemorrhage with no evidence of vasculitis and/or accumulation of immune complexes within lung parenchyma is exhibited. Siderophages in gastric juice content has also been reported to aid diagnosis in a few cases reported in literature. Lung biopsy, however, is the gold standard tool to diagnose IPH [13].

Due to the absence of fundamental diagnostic criteria, therapeutic decisions made against IPH are based on earlier case reports and case studies which favor the corticosteroids use $[1,3,10,14]$. Corticosteroids have shown to reduce the risk of pulmonary fibrosis and relapses of alveolar hemorrhage in patients with IPH, in addition to increasing survival. Immunosuppressive agents, mainly azathioprine, hydroxychloroquine and cyclophosphamide, have shown benefit in patients with unfavorable response to corticosteroids. Azathioprine in combination with corticosteroids has proven to be effective in reducing the risk of relapse [2]. Two cases of lung transplantation in IPH patients have been described in literature, however, IPH recurrence in transplanted lungs precluded any further attempts at transplantation [15].

IPH is characterized by a highly variable prognosis, with acute exacerbations afflicting most patients. A study has demonstrated a 5-year survival rate of $86 \%$ as a ramification of aggressive immunosuppressive therapy. Previous studies had shown mean survival time ranging from 2.5-5 years after diagnosis. Previous studies point towards a better prognosis for adult patients compared to pediatric patients. A systematic review of all adult cases over 15 years delineated mortality in acute stage to be $14 \%$. Other studies have also shown the mortality from IPH to range between $14-29 \%$ and occurring commonly due to acute or chronic respiratory failure, despite immunosuppressive therapy [16]. In our series, one of our three patients died due to acute respiratory failure while being admitted for treatment of IPH.
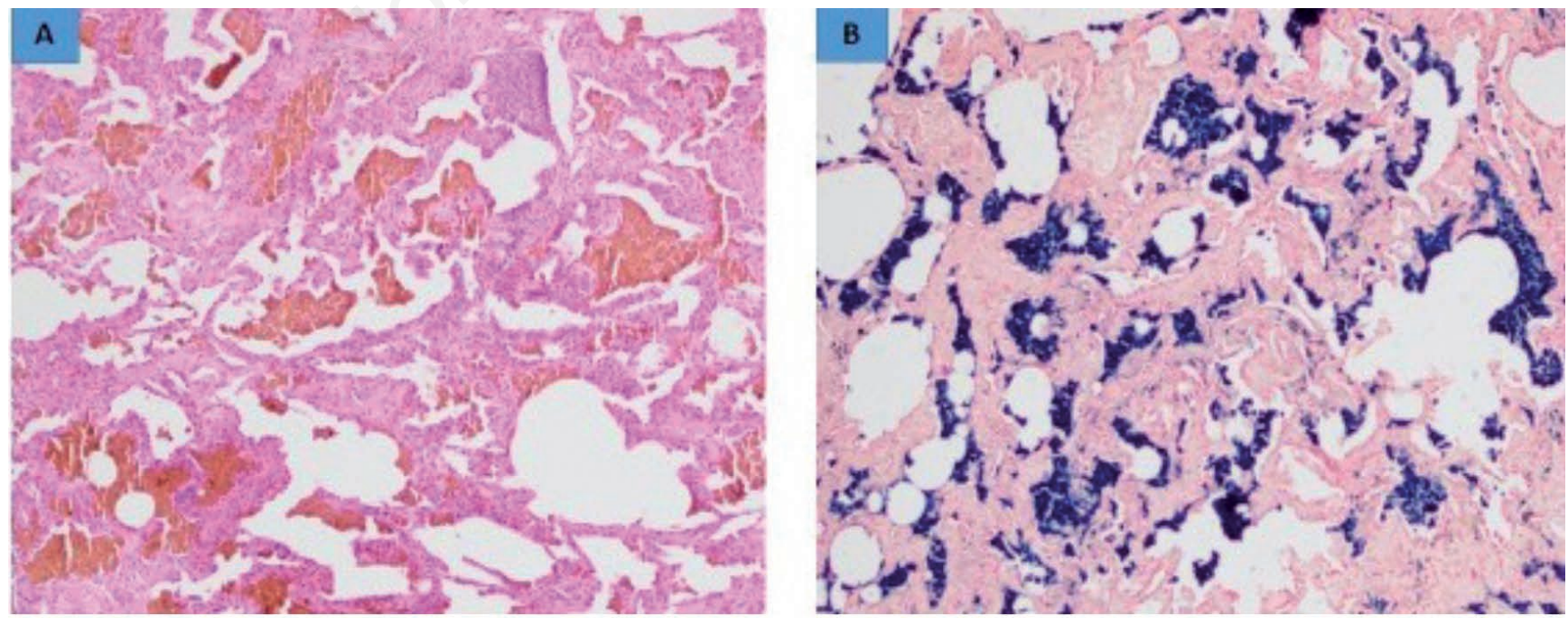

Figure 2. Lung biopsy findings of Patient 3. Haemosiderin laden macrophages in the alveoli (A), highlighted by Perl's Prussian Blue stain for iron (B). 


\section{Conclusions}

Given the severity of the illness, it is imperative for clinicians to consider IPH as an important differential in adult patients who present with diffuse alveolar hemorrhage and also in children with long standing anemia who do not respond to iron therapy. There needs to be a greater awareness amongst physicians regarding IPH so that it can be correctly diagnosed and thus successfully treated.

\section{References}

1. Soergel KH, Sommers SC. Idiopathic pulmonary hemosiderosis and related syndromes. Am J Med 1962;32:499-511.

2. Airaghi L, Ciceri L, Giannini S, et al. Idiopathic pulmonary hemosiderosis in an adult. Favourable response to azathioprine. Monaldi Arch Chest Dis 2001;56:211-3.

3. Bakalli I, Kota L, Sala D, et al. Idiopathic pulmonary hemosiderosis - a diagnostic challenge. Ital J Pediatr 2014;40:35.

4. Moissidis I, Chaidaroon D, Vichyanond P, Bahna SL. Milkinduced pulmonary disease in infants (heiner syndrome). Pediatr Allergy Immunol 2005;16:545-52.

5. Nuesslein TG, Teig N, Rieger CH. Pulmonary haemosiderosis in infants and children. Paediatr Respir Rev 2006;7:45-8.

6. Beckerman RC, Taussig LM, Pinnas JL. Familial idiopathic pulmonary hemosiderosis. Am J Dis Child 1979;133:609-11.
7. Bhatia S, Tullu MS, Vaideeswar P, Lahiri KR. Idiopathic pulmonary hemosiderosis: alveoli are an answer to anemia. J Postgrad Med 2011;57:57-60.

8. Casian A, Jayne D. Current modalities in the diagnosis of pulmonary vasculitis. Expert Opin Med Diagn 2012;6:499-516.

9. Agarwal R, Aggarwal AN, Gupta D. Lane-Hamilton syndrome: simultaneous occurrence of coeliac disease and idiopathic pulmonary hemosiderosis. Intern Med J 2007;37:65-7.

10. Taytard J, Nathan N, de Blic J, et al. New insights into pediatric idiopathic pulmonary hemosiderosis: the French RespiRare((R)) cohort. Orphanet J Rare Di. 2013;8:161.

11. Bulucea $C$ and Sorin D. Idiopathic pulmonary hemosiderosis in children: A romanian experience. Pediatrics 2008;121:S158.

12. Keskin O, Keskin M, Guler E, et al. Unusual presentation: pulmonary hemosiderosis with celiac disease and retinitis pigmentosa in a child. Pediatr Pulmonol 2011;46:820-3.

13. Tzouvelekis A, Ntolios P, Oikonomou A, et al. Idiopathic pulmonary hemosiderosis in adults: a case report and review of the literature. Case Rep Med 2012;2012:267857.

14. Zhang Y, Luo F, Wang N, et al. Clinical characteristics and prognosis of idiopathic pulmonary hemosiderosis in pediatric patients. J Int Med Res 2019;47:293-302.

15. Calabrese F, Giacometti C, Rea F, et al. Recurrence of idiopathic hemosiderosis in a young adult patient after bilateral singlelung transplantation. Transplantation 2002;74:1643-5.

16. Miwa S, Imokawa S, Kato M, et al. Prognosis in adult patients with idiopathic pulmonary hemosiderosis. Intern Med 2011;50:1803-8. 